\title{
Database Management Expert Program for Integrated Evaluation of Non-Invasive and Invasive Results of Coronary Heart Disease
}

\author{
Zs Koszegi, E Balogh, L Balkay, J Varga, L Galuska, Cs Jenei, D Apro, I Edes \\ MHSC Division of Cardiology, University of Debrecen, Hungary
}

\begin{abstract}
The coronary angiography giving the morphological data of epicardiac coronary stenoses and non-invasive tests sometimes even together are insufficient for evaluating the functional consequences of the epicardiac coronary stenoses. However, catheterisation allows us to detect the blunting of coronary flow increase during maximal vasodilatation (coronary flow reserve, CFR, e.g. by Doppler flow wire) and by measuring the translesional pressure gradient by pressure wire (fractional flow reserve, FFR) providing accurate functional assessment. According to this concept the authors have developed a complex cardiac database management program (Holistic Coronary Care, HCC). The database has been built on client-server technology where the central object is the 16-segment polar map. The system contains contextual and graphical elements for a quick data entry. By the assessment of 16 left ventricular segments on a polar map display there is a possibility for direct comparison of the invasive functional and morphologic and the non-invasive functional data. Details measured by pressure or Doppler wire are also stored in the coronary evaluating unit and the coronary segments are rendered to the supplied left ventricular segments. For the correct indication of coronary interventions (from functional and financial point of view) it is important to verify unambiguously the functionally significant coronary stenoses. To the best of our knowledge, the necessity of percutan or surgical intervention can be decided the most accurately by FFR and CFR, furthermore CFR objectively shows the failure of the coronary microvasculature.
\end{abstract}

\section{Introduction}

Coronary angiography is a reliable invasive diagnostic method for visualising the epicardial coronary artery with more than $0.2 \mathrm{~mm}$ in diameter, but it gives limited information about the myocardial resting tissue perfusion and does not assess the coronary flow reserve at all. However, in coronary heart disease (CHD) the current goal for diagnosis is not only to locate the damaged epicardial coronary segments, but also to verify its influence on myocardium. In order to the correct indication of coronary interventions it is important to separate unambiguously the significant and functionally not relevant coronary stenoses. None of the standard or comparative imaging technique itself is capable of comprehensive achievements of all the goals of cardiac imaging. The integration of the data of separated noninvasive and invasive investigations has not been yet fully accomplished. Problems are arising both from the different registration techniques (2D or 3D imaging) and interpretation of anatomical and functional data. The 'holistic' approach is crucial for the correct settlement of the diagnostic and in the therapeutic algorithm of patients with coronary heart disease.

\section{Aims}

To develop a computer program that is enable the integrated evaluation of the results of CHD comparing the anatomical and functional parameters. For this purpose three basic tasks to be solved: 1. to develop a coregistration technique for all the imaging methods, 2. structural database management of the available diagnostic results, 3 . to make possible teleconsultations between doctors from different levels involved in the treatment of the patient (e.g. general practice, hospital and cardiac centre).

\section{Method}

\subsection{Structure of the database}

The authors have constructed a complex cardiac database management program based on Microsoft (MS) Access 2000 managing system as a client program. At low volume hospitals the database server assures the MS Access, but in the advanced configurations it could be either MSQL or MYSQL database using ODBC communication protocol. The present demo version can be downloaded from the http://delfin.unideb.hu/ balkay/HCC/ website. The program consist of two database files with different function; one is the „HCC_base.mdb” database-file, this contains the real data, the other is the „HCC.mde” file, this incudes the forms assuring the correct function, e.g. queries, macros and visual basic codes. The different results can be assessed and integrated. The system contains both contextual and graphical elements for a 
quick data entry. The users can access the data according to predefined permission hierarchy based on their unique account.

\subsection{Data management}

The program was developed to evaluate and visualize the results of the most important invasive and noninvasive cardiological investigation techniques (echocardiography, coronary angiography, coronary flow measurement and SPECT). Data are based uniformly in 16 left ventricular segments therefore can be represented on a polar map (PM) display that is used for the mapping of the heart in nuclear imaging. The diagnostic usefulness of this displaying has been validated [1]. We used a semiquantitative method for polar map registration of the coronary tree [2] focusing on the relation between the supplying coronary branches and the myocardial territories of the 16-segment left ventricular evaluating model. All the registered anatomical and functional data were related to these $16 \mathrm{left}$ ventricular segments allowing the direct comparison and the "holistic" synthesis of the results.

\section{Results}

The database management program stores information about individual CHD patients: anamnestic data, physical status, diagnostic tests and applied drug or interventional therapy during the overall cardiac care. The invasive functional and morphological as well as the non invasive results are displayed on left ventricular polar maps in the evaluating units of the program.

\subsection{Evaluating Units for different cardiac imaging techniques}

\subsubsection{Echocardiography}

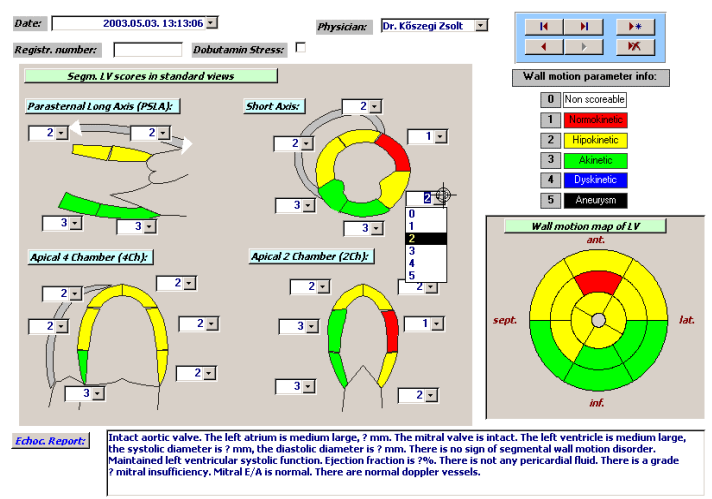

Figure 1. The Echocardiography Evaluating Unit with standard echocardiography views and polar map $\mathbf{. 1}$.

The wall motion polar map is derived from the standard two-dimensional echocardiograms (figure 1.) by use of a 16-segment model [3]. Evaluating substitute scores (1-5) for normokinesia (1), hypokinesia (2), akinesia (3), dyskinesia (4), aneurysm (5) and zero for a non-scorable region. For summarizing the data for the entire left ventricle, the 16 segments scores can be projected for the left ventricle polar map with color codes by the same principles as for the nuclear medicine studies. In the local coordinate system of the heart, from the ("reoriented") 2 and 4 chamber long axis views the 66 examined segments take place along the vertical and the horizontal axis of the polar map. Together with the results of the remaining 4 segments examined from the parasternal view (the short axis slices represents the same segments as examined from the long axis views) all the 16 segments can be projected to the polar map.

\subsubsection{SPECT}

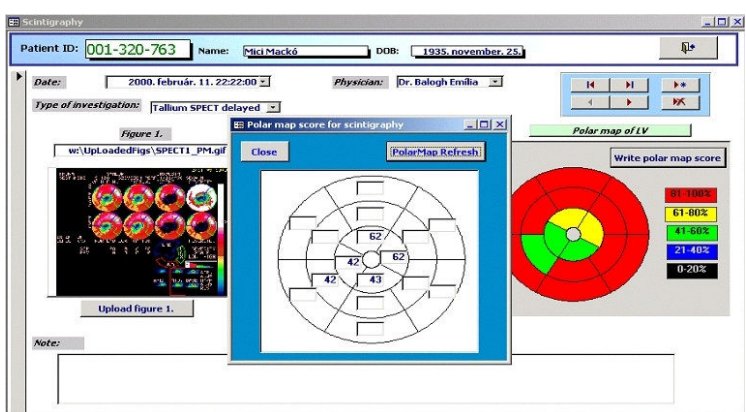

Figure 2. The Isotope Evaluating Unit with polar map

Evaluation of the reoriented slices of the reconstructed images in $3 \mathrm{D}$ is a routine method for the analysis of the scintigraphies. Basically three reoriented slice sets (parallel to the short axis, the horizontal long axis and the vertical long axis of the left ventricle) are displayed for visual evaluation.

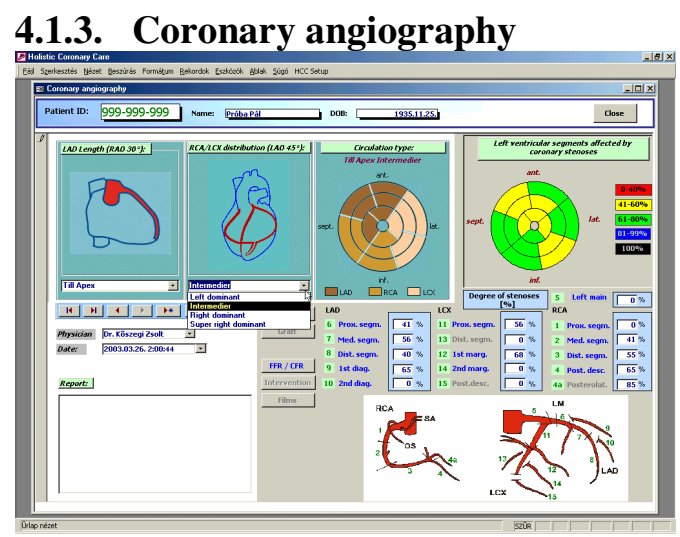

Figure 3. The Coronary Angiography Evaluating Unit The average relative activities of the predefined 16 left ventricular segments can be read easily from the polar 
Usually the commercial software generates the quantitative polar map on the basis of the short axis slices or without this possibility one can enter manually the data (figure 2.) map by usual computer programs.

Adopting the classic anatomic rules and assumptions of the epicardiac surface (of the left ventricle), the 3D characteristics of the coronary artery anatomy can be translated in a 2D-PM display. For comparison, two projections are taken into consideration: the $30^{\circ}$ right anterior oblique (RAO) and the $45^{\circ}$ left anterior oblique (LAO) views. Assessing the coronary angiogram in the standard views (LAO, RAO) the individual coronary artery circulation type can be chosen from twelve variations. The RCA/LCx separation can be determined from the LAO view. The border between the RCA and LCx territories could be assessed by comparing the left and right coronary angiograms from the same view. The position of the lesions can be determined in relation to side branches' hallmarks. The lesion-associated regions are defined as the myocardial bed of a diseased artery distal to the lesion. According to accepted directives [4] the coronary tree is divided into $15+1$ segments. The observed stenoses can be recorded in a chart. The percentage of given stenosis determines the color code of the affected region on the polar map that is calculated automatically.

These parameters are ready to store for each coronary segments.

\subsection{Intracoronary pressure measurement}

You can enter the measured fractional flow reserve (FFR) values into the appropriate segments or register the separate proximal and distal pressure values during resting condition and during vasodilatation. From these values the FFR and the pressure derived CFR (coronary flow reserve) are calculated automatically using the equation on the figure 4. [5].

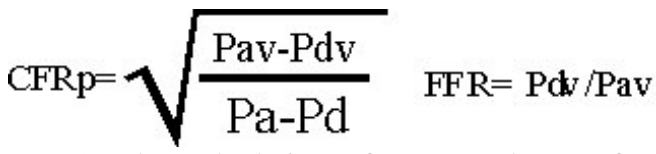

Figure 4. The calculation of CFR and FFR from the measured proximal and distal intracoronary pressure during basal condition and under maximal vasodilatation. Pav: proximal (=aorta) coronary pressure under vasodilatation, Pdv: distal coronary pressure under vasodilatation, $\mathrm{Pa}$ : proximal (=aorta) coronary pressure during basal state, $\mathrm{Pd}$ : distal coronary pressure during basal state

The available CFR values (measured by Doppler wire, TIMI frame count method, contrast echo or PET) can be entered for the 3 main coronary branches and the results will be displayed on a polar map (figure 5.)

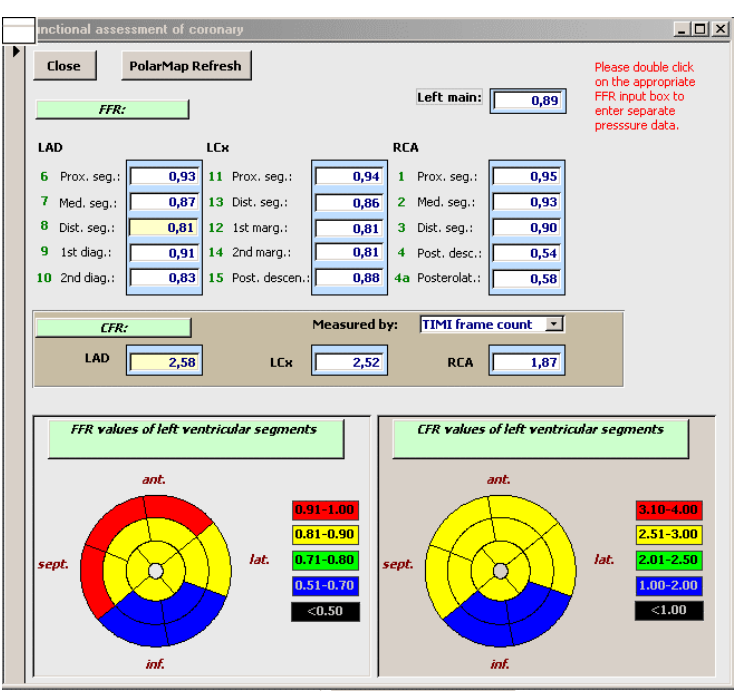

Figure 5.

Polar map interpretation of the measured or calculated FFR and CFR

\subsection{Percutaneous Coronary Intervention (PCI) Register}

At the starting of this register you can choose lesion for the intervention from the detected stenoses had been input on the Coronary Angiography Evaluating Unit in the previous window. It contains the following data relating to PCI: nature of the stenosis (native, graft, stent), balloon and stent parameters, degree of the residual stenosis, type of complication.

After input of the result of the intervention the polar map will be refreshed according to the achieved residual stenosis.

\subsection{Data integration}

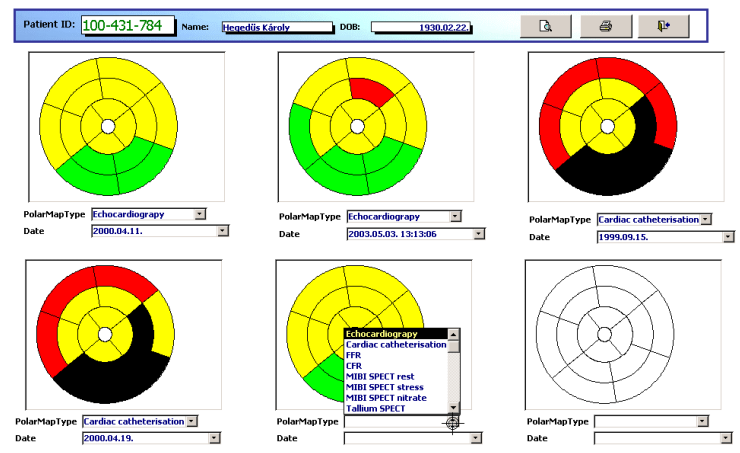

Figure 6. Polar Map Summary Unit

The Polar Map Summary unit assures the possibility to compare six different reports on the 16-segment polar map display. These direct comparisons on the one hand 
enable us to integrate information of different imaging technique for careful evaluation of the functional consequence of the coronary artery disease. On the other hand it helps to evaluate the follow up of the patient in case of serial investigations (from the same type of technique). The reports assigned by different visualization methods are converted to standard color codes (red-yellow-green-blue-black) and related all to 16 left ventricular myocardial segments.

A DICOM viewer module is under development in order to evaluate the archived examinations for every imaging technique. The access of the original images enable us to teleconsultate the case.

\section{Conclusion}

Variations in coronary anatomy have to be always considered to associate the affected coronary region with the supplying coronary artery. This requires (at least theoretically) 3D registration of both the coronary artery system and the imaged left ventricle to the same coordinate system. Despite the fact that accurate algorithms have been worked for assessing the 3D structure of the coronary tree [6-7], for the time being no software has been introduced for clinical practice in this field.

This program was developed for direct comparison of tomographic imaging and echocardiography with the angiogram on the polar map display in order to assess the functional consequences of coronary artery stenosis. The careful evaluation of the functional consequence of the coronary artery disease helps us to indicate accurately the necessity of percutan or surgical intervention on a certain lesion or coronary branch.

The database management program simplifies the evaluation and integration of many important cardiological imaging methods, objectifies the clinical decision-making and also helps us in the assessment of the effect of the pharmaceutical or revascularistion treatment during the follow up.

Furthermore, the program can be upgraded to make it suitable for both ranking the patients on the waiting list of interventions more objectively as well as for modernization of the distinct follow-up procedures.

\section{References}

[1] Milan E; Zoccarato O; Terzi A; Ettori F; Leonzi O, Niccoli L; Giubbini R. Technetium-99m-sestamibi SPECT to detect restenosis afret successful percutaneous coronary angioplasty. J Nucl Med. 1996;37(8): 1300-5

[2] Koszegi Z., Maes A., Piessens J.,Van de Werf F., Mortelmans L.: Segmental comparison between coronary angiography and PET reveals low predictive value of epicardial flow for viability. European Heart Journal, 1998; 19: 959-967

[3] Schiller NB; Shah PM; Crawford M; DeMaria A; Devereux R; Feigenbaum H; Gutgesell H; Reichek N; SahnD; Schnittger-I; et-al: Recommendations for quantitation of the left ventricle by two-dimensional echocardiography. American Society of Echocardiography Committee on Standards, Subcommittee on Quantitation of Two-Dimensional Echocardiograms. J Am Soc Echocardiogr. 1989; 2(5): 358-367.

[4] James TN; Bruschke AV; Bothig S; Dodu SR; Gil JF; Kawamura-K; Paulin-SJ; Piessens-J: Report of WHO/ISFC Task Force on Nomenclature of Coronary Arteriograms. Circulation. 1986; 74(2): 451A-455A

[5] E. Shalman, C. Barak, E. Dgany, H. Noskowitcz, S. Einav, M. Rosenfeld: Pressure-based simultaneous CFR and FFR measurements: understanding the physiology of a stenosed vessel Computers in Biology and Medicine 2001; 31: 353363

[6] Dodge JT Jr; Brown Bg; Bolson EL; Dodge HT. Intrathoracic spatial location of specified coronary segments on the normal human heart. Applications in quantitiative arteriography, assessment of regional risk and contraction, and anatomic display. Circulation, 1988; 78 : 1167-80

[7] Solzbach U; Oser U; Rombach M; Wollschlager H; Just H. Optimum angiographic visualization of coronary segments using computer-aided 3D reconstruction from biplane views. Computer Biomed Res 1994; 27(3): 178-98

Address for correspondence:

Zsolt Koszegi M.D., PhD

MHSC Division of Cardiology

University of Debrecen

4004 Debrecen, P.O.B.

Hungary

e-mail: koszegi@jaguar.dote.hu 